\title{
Consumo de Álcool e a sua Associação com a Atividade Física em Adolescentes Escolares
}

\author{
Alcohol Consumption and its Association with Physical Activity \\ Among School Adolescents
}

\author{
Mayra Ruana de Alencar Gomes ${ }^{1}$ \\ Alaine Sousa Lima² \\ Rachel Mola ${ }^{3}$ \\ Rodrigo Cappato de Araújo ${ }^{4,5}$ \\ Ana Carolina Rodarti Pitangui ${ }^{5,6}$
}

\section{RESUMO}

Objetivo: Verificar a prevalência do consumo de álcool e a sua associação com a atividade física em adolescentes escolares de um município do interior de Pernambuco. Metodologia: Trata-se de um estudo epidemiológico, de base escolar, de caráter descritivo e correlacional e com delineamento transversal, realizado com adolescentes com idade entre 12 a 17 anos, matriculados no ensino fundamental e médio de escolas da rede pública do Município de Petrolina. Os adolescentes foram submetidos aos inquéritos sociodemográfico e sobre os comportamentos de risco que consistiu no questionário Youth risk behavior survey (YRBS). As prevalências e as possíveis associações foram analisadas em modelos múltiplos de regressão logística binária, entre as variáveis dependentes, o consumo de álcool e o episódio de bebedeira nos últimos 30 dias com as demais variáveis. A razão de chances (OR) e os intervalos de confiança de $95 \%$ foram calculados. Resultados: Foram incluídos na análise final 1179 adolescentes, 50,9\% relataram já ter feito uso de álcool alguma vez na vida, destes, $73 \%$ tiveram o primeiro contato com o álcool antes dos 15 anos, 49,6\% consumiram nos últimos 30 dias e $34,3 \%$ tiveram episódios de embriaguez. Apenas, $16,4 \%$ dos participantes referiram ser fisicamente ativos. A atividade física demonstrou associação positiva com o consumo atual de álcool (OR=1,67 [1,11-2,52]) e com os episódios de bebedeira (OR=1,88 [1,03-3,33]). Conclusão: Pode-se concluir que as taxas de consumo de álcool e de bebedeira foram elevadas e apresentaram associação positiva com a prática de atividade física atual, tendo os adolescentes ativos mais chances de consumir álcool.

\section{DESCRITORES}

Adolescente. Comportamento de Risco. Bebidas Alcoólicas. Bebedeira. Atividade Física.

\begin{abstract}
Objective: To verify the prevalence of alcohol consumption and its association with physical activity in school adolescents in a city in the interior of Pernambuco. Methodology: This is an epidemiological, school-based, descriptive and correlational study with a cross-sectional design, conducted with adolescents aged between 12 and 17 years old, enrolled in elementary and high school in public schools in the city of Petrolina. The adolescents were submitted to sociodemographic and risk behavior surveys, which consisted of the Youth Risk Behavior Survey (YRBS) questionnaire. The prevalence and possible associations were analyzed in multiple models of binary logistic regression, between the dependent variables alcohol consumption and binge-drinking episode in the last 30 days with the other variables. The odds ratio (OR) and the $95 \%$ confidence intervals were calculated. Results: 1179 adolescents were included in the final analysis, $50.9 \%$ reported having used alcohol once in their lives, of which $73 \%$ had their first contact with alcohol before the age of $15,49.6 \%$ had consumed it in the last 30 days and $34.3 \%$ had episodes of drunkenness. Only $16.4 \%$ of the participants reported being physically active. Physical activity showed a positive association with current alcohol consumption $(\mathrm{OR}=1.67[1.11-2.52])$ and binge drinking episodes $(\mathrm{OR}=1.88$ [1.03 - 3.33]). Conclusion: It can be concluded that the rates of alcohol consumption and drinking were high and showed a positive association with the practice of current physical activity, with active adolescents more likely to consume alcohol.
\end{abstract}

\section{DESCRIPTORS}

Adolescents. Risk Behavior. Alcoholic Beverages. Binge Drinking. Physical Activity.

1. Doutoranda do Programa de Pós-Graduação em Saúde da Criança e do Adolescente - Universidade Federal de Pernambuco - UFPE, Recife, Pernambuco, Brasil.

2. Doutoranda do Programa de Pós-Graduação em Ciências da Saúde - Universidade de Pernambuco - UPE, Recife, Pernambuco, Brasil,

3. Professora do curso de Enfermagem da Universidade de Pernambuco - UPE, Petrolina, Pernambuco, Brasil.

4. Professor do Programa Associado de Pós-Graduação em Educação Física - UPE/UFPB, Recife, Pernambuco, Brasil.

5. Professor do curso de Fisioterapia da Universidade de Pernambuco - UPE, Petrolina, Pernambuco, Brasil

6. Professora do Programa Associado de Pós-Graduação em Enfermagem UPE/UEPB, Recife, Pernambuco, Brasil. 
E ntre as substâncias psicoativas, o álcool é a droga mais consumida no mundo e no Brasil pelos adolescentes ${ }^{1}$. Isso ocorre devido ao fato de ser uma droga lícita facilmente disponível, amplamente aceita pela sociedade e que produz sensações prazerosas ${ }^{2,3}$. O uso do álcool apresenta inúmeras consequências físicas, sociais e psicológicas para os indivíduos, sendo associado a diferentes condições de saúde e à morte ${ }^{4,5}$. Especificamente ao adolescente, o uso do álcool prejudica a neurogênese, comprometendo o desenvolvimento cerebral e repercutindo em danos às funções neurocognitivas ${ }^{6-8}$.

Segundo o último Levantamento Nacional sobre o Consumo de Drogas Psicoativas elaborado pelo Centro Brasileiro de Informações Sobre Drogas Psicotrópicas (CEBRID), realizado com adolescentes escolares de 27 capitais brasileiras, $60,5 \%$ dos escolares relataram ter usado álcool alguma vez na vida; $42,4 \%$ no último ano; $21,1 \%$ nos últimos 30 dias; $2,7 \%$ uso frequente, ou seja, seis ou mais vezes no mês; e 1,6\% uso pesado, isto é, 20 ou mais vezes no último mês ${ }^{9}$.

Além da alta prevalência do uso de bebidas alcoólicas pelos adolescentes, outros dois fatores são importantes, a idade de início e o padrão de consumo do álcool. Alguns estudos têm evidenciado que o contato do adolescente com o álcool tem ocorrido cada vez mais cedo ${ }^{7,10}$. Por sua vez, os adolescentes constituem um grupo significativamente vulnerável para apresentarem problemas relacionados ao álcool ${ }^{11}$.

Múltiplos fatores estão envolvidos no desenvolvimento do comportamento de uso de substâncias como o álcool, ${ }^{12,13}$ tais como o sexo masculino, maior idade, ${ }^{13-15}$ maior renda familiar; relação ruim com os pais, sentimento de tristeza e solidão; ${ }^{16}$ religiosidade, uso pelos pais e amigos; prática esportiva e atividade física no lazer ${ }^{17,18}$. Dentre os fatores investigados, a prática da atividade física (AF) merece destaque pois apresenta função importante na saúde e no desenvolvimento dos adolescentes. Conduto, os achados da literatura são conflitantes em relação ao nível de atividade física e consumo de álcool ${ }^{17,18}$.

A AF tem sido discutida como um fator protetor para diversos fatores de risco à saúde, e de fato, estudos com populações europeias têm demonstrado que a prática de atividade física apresenta associação inversa com o consumo de álcool ${ }^{19,20}$, porém dados de adolescentes norte americanos ${ }^{21}$ e de revisão sistemática ${ }^{22}$ demonstraram uma relação positiva entre AF e consumo de álcool.

Conhecer a prevalência desse consumo e verificar os fatores associados é de grande importância para que ocorram melhorias no planejamento de programas de saúde pública que tenham como propósito diminuir as consequências negativas relacionadas ao uso dessa substância. Assim, compreender melhor a relação entre a atividade física e o consumo de álcool na população adolescente é importante, pois oferece subsídios no planejamento de intervenções e norteia os programas de prevenção. Com base neste contexto, o objetivo deste estudo foi verificar a associação entre o consumo de álcool com atividade física e fatores sociodemográficos em adolescentes escolares do município de Petrolina-PE. 


\section{METODOLOGIA}

Estudo transversal, de base escolar, de caráter descritivo e correlacional, realizado no período de março a julho de 2014 , nas instituições de ensino fundamental e médio da rede pública (estadual), situadas no município de Petrolina - PE. A população estudada foi constituída por adolescentes com idade entre 12 e 17 anos, estudantes das instituições de ensino supracitadas, que consentiram em participar da pesquisa e que, estavam com o Termo de Consentimento Livre e Esclarecido-TCLE autorizado pelo responsável legal.

A pesquisa obedeceu a todos os preceitos da Resolução 466/12 do ConseIho Nacional de Saúde (CNS), e as orientações do ECA, sendo aprovado pelo Comitê de Ética em Pesquisa da Universidade de Pernambuco sob protocolo CEP/UPE $n^{\circ}$ 24288213.2.0000.5207 e parecer $n^{\circ} 521.340$.

Para quantificação da amostra utilizou-se o programa WinPepi, considerou-se uma população de 25.635 estudantes, intervalo de confiança de $95 \%$; erro máximo tolerável de 5 pontos percentuais; perda amostral de $20 \%$; e por se tratar de estudo abrangendo a análise de diferentes comportamentos de risco e com diferentes frequências de ocorrência, a prevalência estimada usada foi de $50 \%$, totalizando o quantitativo de 474 adolescentes. Esperou-se que as proporções de meninos/meninas da manhã, tarde e integral fossem equivalentes da população favorecendo a representatividade na amostra final. Para tanto, foi realizada a multiplicação do tamanho mínimo da amostra por 2.0 (efeito do delineamento de amostragem), totalizando 948 adolescentes. Considerando um número mínimo de 17 alunos por turma, avaliou-se 1.226 adolescentes.

O instrumento da coleta de dados foi constituído por inquérito sociodemográfico, as questões foram apresentadas de modo esquemático, por meio do recurso de questões de múltipla escolha, objetivando facilitar as respostas. O participante foi informado a escolher apenas uma das alternativas. Para avaliação dos comportamentos de risco foi empregada a versão brasileira do questionário Youth Risk Behavior Survey (YRBS), composto por 87 questões relacionadas a seis categorias de comportamentos de risco a saúde em adolescentes e jovens: 1) lesões não-intencionais e violência; 2) uso de tabaco; 3) consumo de bebidas alcoólicas e outras drogas; 4) comportamento sexual voltado à gravidez indesejada e às doenças sexualmente transmissíveis; 5) hábitos alimentares; e 6) prática de atividade física ${ }^{23}$. No presente estudo foram analisados os domínios de consumo de bebidas alcoólicas e atividade física.

A tabulação dos dados foi realizada no programa Microsoft Excel utilizando o procedimento de dupla digitação. Os dados foram analisados utilizando o programa SPSS, versão 20.0 (SPSS Inc., Chicago, IL, Estados Unidos da América). A análise descritiva foi usada para apresentação dos dados, no caso das variáveis categóricas incluiu a distribuição das frequências absolutas e relativas, e para as variáveis numéricas foram calculados os valores de tendência central e dispersão de acordo com a distribuição dos dados, que foi verificada por meio do teste Kolmogorov Smirnov.

Foram calculadas as associações en- 
tre as variáveis dependentes e cada variável independente, por meio do teste Qui-Quadrado. Nesta análise, foi utilizada a estimativa da razão de chances (Odds Ratio, OR) e intervalos de confiança de 95\% [IC 95\%] para expressar o grau de associação entre a variável dependente e independentes. As variáveis independentes que apresentaram um nível de significância de $20 \%$ foram selecionadas para análise de regressão logística. Em seguida, foi utilizado o diagnóstico de colinearidade para testar se existia multicolinearidade entre as variáveis independentes. Nenhum índice de tolerância (todos < 1) e nem valores VIF (todos $<2$ ) indicaram a presença de multicolinearidade

Vários tipos de modelagens foram testados a fim de se obter um modelo válido e que apresentasse a melhor qualidade de ajuste. Para essas análises foram considerados os Testes de coeficientes de modelo Omnibus, teste de Nagelkerke R Square e o teste de Hosmer and Lemeshow.

Por fim, foram executados modelos de regressão logística binária por meio do método condicional retroceder, para as variáveis dependentes: consumo atual de álcool e bebedeira. Todos os testes foram bicaudais, e em todas as análises foi adotado um nível de significância de $p<0.05$.

\section{RESULTADOS}

Do total de adolescentes avaliados, 1179 foram incluídos na análise final dos dados, portanto foram excluídos 47 voluntários da pesquisa devido ao não preenchimento adequado das informações. Ao analisar os dados verificou-se que a idade dos adoles- centes avaliados apresentou mediana de 15 anos, 37,4\% apresentavam idade entre $16-17$ anos, $56,4 \%$ do sexo feminino, $55 \%$ se autodeclaram de cor parda, $50,3 \%$ se dizem católicos, $66,5 \%$ tem renda familiar ente 1-2 SM e $94,7 \%$ declaram estado civil solteiro.

Do total dos indivíduos analisados, 50,9\% [IC 95\% 48,0 - 53,8] relataram já ter feito uso de bebidas alcoólicas alguma vez na vida. Verificou-se que $83,6 \% \quad(n=967)$ dos adolescentes foram classificados como insuficientemente ativos. Dentre os meninos $24,6 \%(n=124)$ eram ativos e $75,4 \%(n=380)$ insuficientemente ativos, enquanto entre as meninas $10,1 \%(n=66)$ eram ativas e $89,9 \%$ $(n=587)$ insuficientemente ativas.

A Tabela 1 demonstra a associação entre o consumo atual de bebidas alcoólicas com as variáveis independentes estudadas nos adolescentes. A análise ajustada revelou que a variável religião se mostrou um fator que diminui as chances de uso de álcool, e que atividade física atual e experimentação de álcool aumenta as chances de uso.

A Tabela 2 apresenta a associação entre bebedeira nos últimos 30 dias e as variáveis independentes estudadas nos adolescentes. A análise ajustada mostrou que as variáveis idade, atividade física atual, consumo atual e experimentação de álcool permaneceram no modelo final. $O$ nível de atividade física, consumo atual e experimentação de álcool se demonstraram fatores que aumentam as chances de envolvimento dos adolescentes em episódios de bebedeira. A fator idade não apresentou associação com a bebedeira no modelo final. 
Tabela 1. Associação entre o consumo atual de bebidas alcoólicas com as variáveis independentes estudadas nos adolescentes. Petrolina-PE, 2014

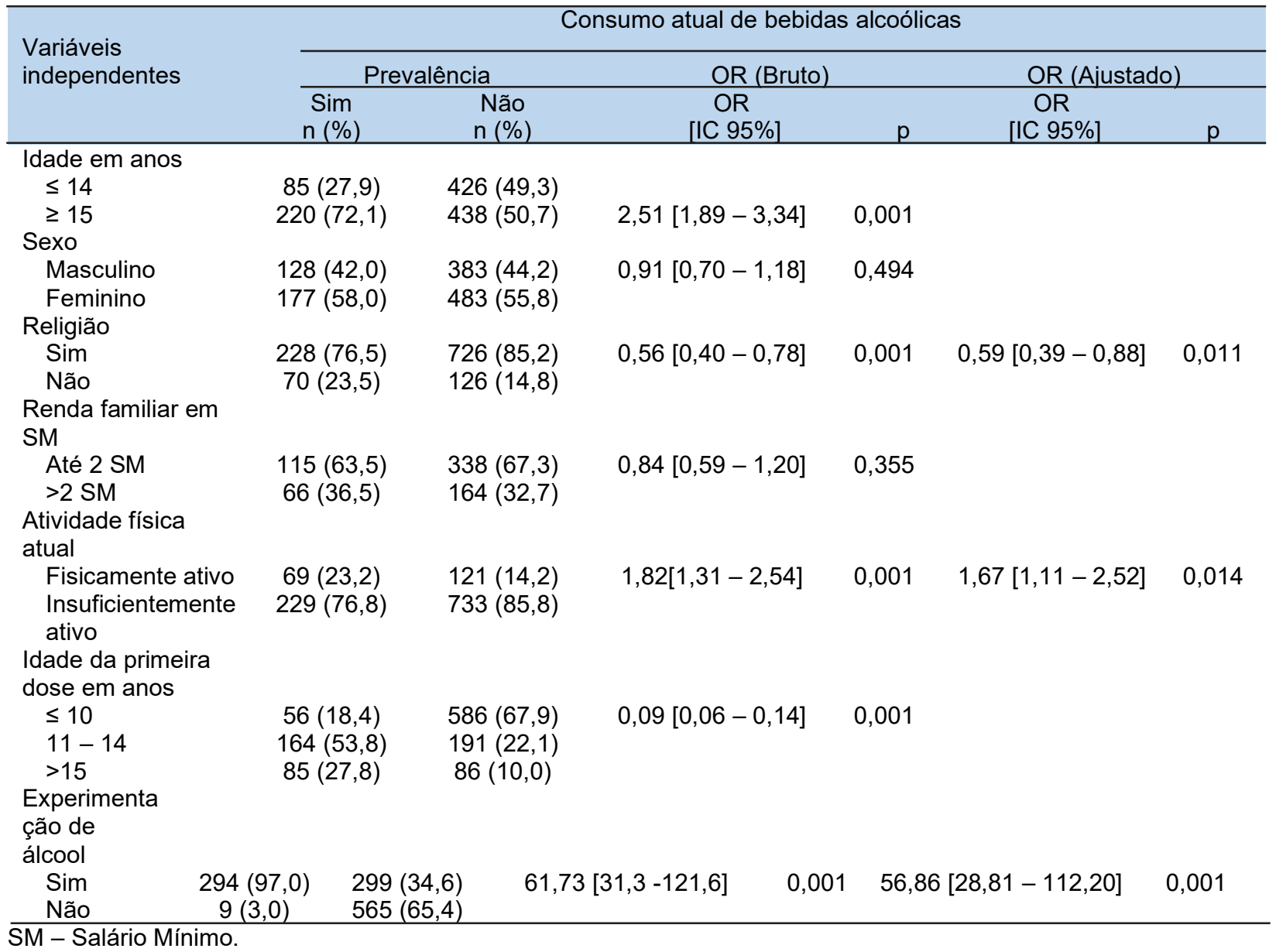

\section{DISCUSSÃO}

Referente ao consumo de bebidas alcoólicas, mais da metade dos adolescentes relatou já ter feito uso de álcool alguma vez na vida, corroborando achados de estudos prévios. ${ }^{7,9}$ Essa alta prevalência se deve a múltiplos fatores, entre os quais estão a própria adolescência, que é um período marcado pela impulsividade e experimentação ${ }^{24}$ e as expectativas que os adolescentes constroem em relação ao consumo do álcool, no sentido que essa substância irá proporcionar uma maior autoconfiança, interação social, desinibição e atratividade física. Além disso, diferentes formas de mídia contribuem na motivação do adolescente em consumir precocemente o álcool, pois enfatizam essas expectativas ${ }^{25,26}$. Entre aqueles adolescentes que já fizeram uso de álcool alguma vez na vida, foi constatado que quase metade $[49,6 \%]$ referiu consumo atual. No entanto, este resultado foi superior ao encontrado por Bezerra et al. ${ }^{27}$ [30,4\%], que analisou somente estudantes do 
Tabela 2. Associação entre bebedeira com as variáveis independentes estudadas nos adolescentes. Petrolina-PE, 2014

\begin{tabular}{|c|c|c|c|c|c|c|}
\hline \multirow{4}{*}{$\begin{array}{l}\text { Variáveis } \\
\text { independentes }\end{array}$} & \multicolumn{6}{|c|}{ Bebedeira } \\
\hline & \multicolumn{2}{|c|}{ Prevalence } & \multicolumn{2}{|l|}{ OR (crude) } & \multicolumn{2}{|l|}{ OR (Adjusted) } \\
\hline & Sim & Não & OR & & OR & \\
\hline & $n(\%)$ & $\mathrm{n}(\%)$ & [IC 95\%] & $p$ & [IC 95\%] & $p$ \\
\hline \multicolumn{7}{|l|}{ Idade em anos } \\
\hline$\leq 14$ & $53(25,5)$ & $457(47,6)$ & & & & \\
\hline$\geq 15$ & $155(75,5)$ & $503(52,4)$ & $2,66[1,90-3,72]$ & 0,001 & $1,63[0,97-2,73]$ & 0,065 \\
\hline \multicolumn{7}{|l|}{ Sexo } \\
\hline Masculino & $96(45,9)$ & $415(43,1)$ & $1,12[0,83-1,51]$ & 0,461 & & \\
\hline Feminino & $113(54,1)$ & $547(56,9)$ & & & & \\
\hline \multicolumn{7}{|l|}{ Religião } \\
\hline Sim & $152(74,5)$ & $802(84,8)$ & $0,53[0,37-0,75]$ & 0,001 & & \\
\hline Não & $52(25,5)$ & $144(15,2)$ & & & & \\
\hline \multicolumn{7}{|l|}{$\begin{array}{l}\text { Renda familiar } \\
\text { em SM }\end{array}$} \\
\hline $\begin{array}{l}\text { Até } 2 \mathrm{SM} \\
>2 \mathrm{SM}\end{array}$ & $\begin{array}{l}80(67,8) \\
38(32,2)\end{array}$ & $\begin{array}{l}373(66,4) \\
189(33,6)\end{array}$ & $1,07[0,70-1,63]$ & 0,765 & & \\
\hline \multicolumn{7}{|l|}{$\begin{array}{l}\text { Atividade física } \\
\text { atual }\end{array}$} \\
\hline $\begin{array}{l}\text { Fisicamente } \\
\text { ativo }\end{array}$ & $52(25,9)$ & $137(14,4)$ & $2,07[1,44-2,98]$ & 0,001 & $1,88[1,03-3,33]$ & 0,030 \\
\hline $\begin{array}{l}\text { Insuficiente e } \\
\text { ativo }\end{array}$ & $149(74,1)$ & $814(85,6)$ & & & & \\
\hline \multicolumn{7}{|l|}{$\begin{array}{l}\text { Idade da } \\
\text { primeira dose } \\
\text { em anos }\end{array}$} \\
\hline$\leq 10$ anos & $40(19,2)$ & $600(62,6)$ & $0,16[0,10-0,26]$ & 0,001 & & \\
\hline $11-14$ & $119(56,9)$ & $237(24,7)$ & & & & \\
\hline$>15$ & $50(23,9)$ & $122(12,7)$ & & & & \\
\hline \multicolumn{7}{|l|}{$\begin{array}{l}\text { Consumo atual } \\
\text { de álcool }\end{array}$} \\
\hline Sim & $190(91,8)$ & $115(12,0)$ & $82,12[48,19-139,95]$ & 0,001 & $37,91[19,80-72,5$ & 0,001 \\
\hline Não & $17(8,2)$ & $845(88,0)$ & & & & \\
\hline \multicolumn{7}{|l|}{$\begin{array}{l}\text { Experimentação } \\
\text { de álcool }\end{array}$} \\
\hline Sim & $204(98,1)$ & $391(40,8)$ & $74,09[27,41-200,96]$ & 0,001 & $5,82[1,92-17,62]$ & 0,002 \\
\hline Não & $4(1,9)$ & $568(59,2)$ & & & & \\
\hline
\end{tabular}

ensino médio da rede pública estadual de Pernambuco, com faixa etária entre 14 e 19 anos. Os resultados da PeNSE em 2009 [27,3\%] e em $2012[26,8 \%]^{6}$, assim como o último Levantamento Nacional sobre o Consumo de Drogas Psicoativas $[21,1 \%]^{9}$ também demonstraram taxas menores ao presente estudo. Tal fato pode ser justificado devido a presente pesquisa ser realizada com adolescentes de um município do interior de Pernambuco, no qual as opções de lazer são mais restritas quando comparado as das capitais. Além do fator demográfico, devem ser levados em consideração os fatores socioculturais que também produzem particularidades que podem influenciar o estilo de vida e o modo de agir dos adolescentes que moram em cidades do interior, incluindo sua relação com o uso de bebidas alcoólica? ${ }^{7}$.

Além da alta prevalência do uso de 
bebidas alcoólicas pelos adolescentes, outros dois fatores são importantes, como a idade de início e o padrão de consumo do álcool. Em relação à idade de iniciação ao consumo do álcool o presente estudo observou que a maior parte dos adolescentes relataram ter tido o primeiro contato com essa substância entre os 13 e 14 anos de idade. Contudo, alguns estudos apontam a iniciação de bebidas alcóolicas ainda mais cedo, em média aos 11 anos $^{28}$. Esse dado é preocupante, pois os adolescentes constituem um grupo significativamente vulnerável para apresentarem problemas relacionados ao álcool, sendo que o consumo dessa substância em idades precoces apresenta-se como fator preditor relevante para o desenvolvimento de problemas a longo prazo, tais como a dependência na vida adulta. ${ }^{11}$

Em relação aos níveis de atividade física, foi observado no presente estudo que $83,6 \%$ dos adolescentes analisados se mostraram insuficientemente ativos, corroborando o estudo realizado por Hallal et al. ${ }^{29}$ que verificou que aproximadamente $80 \%$ dos adolescentes de 13 a 15 anos no mundo não alcançam as recomendações de prática de atividade física sugeridas pela OMS. Contudo, essa taxa foi superior a de outros estudos nacionais ${ }^{15,27,30}$.

Os baixos níveis de atividade física entre adolescentes têm sido relacionados ao aumento do tempo de comportamento sedentários, como também à redução da opção de lazer ativo devido ao crescimento da violência urbana ${ }^{29}$. No entanto, a prática regular de atividade física em adolescentes e jovens é imprescindível, já que influencia no desenvolvimento físico e cognitivo, além de aumentar as possibilidades de se tornarem adultos ativos $^{29,30}$.

Tem-se observado que comportamentos de risco como o consumo abusivo de bebidas alcoólicas e níveis insuficientes de atividade física, medidos na infância e adolescência constituem fatores de risco para DCNT, tais como doenças cardiovasculares, respiratórias, o diabetes e o câncer, na idade adulta, isso porque a precocidade na exposição a esses fatores pode resultar em um maior acúmulo ao longo da vida, sendo assim, em um maior risco de DCNT6.

Foi constatado no presente estudo que os adolescentes que já experimentaram bebidas alcoólicas na vida tem 56 vezes mais chances de consumirem atualmente e 5,8 de apresentarem episódios de bebedeira. Sabe-se que mesmo o uso experimental do álcool pode trazer prejuízos à saúde do adolescente e levar a alterações de conduta, visto que os danos provocados pelo consumo dessa substância em adolescentes distinguem-se dos evidenciados em indivíduos adultos, tanto em decorrência das características psicossociais próprias dessa fase da vida, tais como a sensação de onipotência e o ato de desafiar regras, ${ }^{4}$ como também por fatores neuroquímicos específicos em virtude do amadurecimento cerebral que está em conclusão nesse período 4 .

O principal resultado neste estudo foi a identificação de que a prática de atividade física atual esteve associada positivamente ao consumo atual e exagerado de bebidas alcoólicas. Assim, foi observado que os adolescentes que praticam atividade física atual tiveram aproximadamente 1,7 vezes mais chances de consumo atual de ácool, da mesma forma a 
pratica de atividade física aumenta em 1,9 vezes mais chances episódios de bebedeira. Tal achado pode ser justificado devido às atividades físicas de final semana serem realizadas geralmente em eventos sociais e de lazer, no qual o consumo de bebidas alcoólicas está comumente ligado ${ }^{27}$. Vale destacar, que esse resultado alerta para o reconhecimento de grupos de riscos com o objetivo de prevenir o consumo precoce e exagerado do álcool, e não tem o intuito de deixar de incentivar a realização de atividade física, visto os inúmeros benefícios que repercute na saúde ${ }^{29,30}$. Assim, entende-se que a prática de atividade física é um recurso de socialização e o fato de estar ligado a um maior consumo de bebidas alcoólicas é por este motivo ${ }^{27}$.

Algumas limitações devem ser mencionadas em relação ao presente estudo, como o fato de o instrumento utilizado ser baseado em respostas auto relatadas, podendo desta forma apresentar vieses de informação. Ainda, por se tratar de uma pesquisa transversal, torna-se impossível determinar o efeito causal dos comportamentos de risco avaliados, o que seria melhor captado em estudos longitudinais, mas possibilita constatar a existência ou não da relação entre as variáveis estudadas. Também cabe salientar que essa pesquisa foi aplicada apenas em estudantes do ensino público que se encontravam presentes em sala de aula, sendo assim, os resultados encontrados podem não refletir o que ocorre com estudantes de escolas particulares.
Por fim, sugere-se a realização de pesquisas futuras que tenham delineamento longitudinal, que analisem adolescentes e jovens de diferentes localidades e provenientes tanto de escolas públicas como privadas. A elaboração de estudos futuros irá contribuir para enriquecer as informações acerca do tema e ajudarão no desenvolvimento de campanhas e programas de prevenção direcionados ao público de risco identificado.

\section{CONCLUSÃO}

Pode-se concluir que o consumo de álcool esteve associado positivamente a atividade física nos adolescentes escolares avaliados. Em relação ao consumo atual, os adolescentes que referiram possuir religião tiveram menos chances de consumir bebidas alcoólicas nos últimos trinta dias. $\mathrm{E}$ aqueles que experimentaram álcool alguma vez na vida e que relataram ser fisicamente ativos foram mais propensos a consumir essa substância atualmente. Os voluntários que já experimentaram e consumiram álcool atualmente tiveram mais chances de apresentar bebedeira.

Por fim, observou-se que as taxas de consumo de álcool na vida, nos últimos trinta dias e episódios de bebedeira foram elevadas, assim como a prevalência em idades precoces. Do mesmo modo, a prevalência de indivíduos insuficientemente ativos também se apresentaram altas. 


\section{REFERÊNCIAS}

1. Arantes LFR. Binge drinking: um estudo bibliométrico (1999-2010) dos artigos publicados na base de dados SciELO. Estud Psicol (Campinas). 2012; 29(2):253-257.

2. Cardoso FM. et al. Fatores associados à prática do binge drinking entre estudantes da área da saúde. Rev CEFAC. 2015; 17(2):475-484.

3. Romano M, Duailibi S, Pinsky I, Laranjeira R. Alcohol purchase survey by adolescents in two cities of State of São Paulo, Southeastern Brazil. Rev Saúde Pública. 2007; 41(4):495-501.

4. Pechansky F, Szobot CM, Scivoletto S. Alcohol use among adolescents: concepts, epidemiological characteristics and etiopatogenic factors. Rev bras psiquiatr. 2004; 26(Suppl 1):S14-7.

5. Peden AE, Franklin RC, Leggat PA. Breathalysing and surveying river users in Australia to understand alcohol consumption and attitudes toward drowning risk. BMC Public Health. 2018; 18(1):1393.

6. Malta DC. et al. Alcohol consumption among Brazilian Adolescents according to the National Adolescent Schoolbased Health Survey (PeNSE 2012). Rev Bras Epidemiol. 2014; 17(1):203-14.

7. Reis TG, Oliveira LCM. Pattern of alcohol consumption and associated factors among adolescents students of public schools in an inner city in Brazil. Rev Bras Epidemiol. 2015; 18(1):13-24.

8. Tavares ER, Silva-Gotay A, Riad WV, Bengston L, Richardson HN. Sex Differences in the Effect of Alcohol Drinking on Myelinated Axons in the Anterior Cingulate Cortex of Adolescent Rats. Brain Sci. 2019; 9(7):167.

9. CEBRID. VI Levantamento Nacional sobre o Consumo de Drogas Psicotrópicas entre Estudantes do Ensino Fundamental e Médio das Redes Pública e Privada de Ensino nas 27 Capitais Brasileiras.

10. Campos JADB, Almeida JC, Garcia PPNS, Faria JB. Consumo de álcool entre estudantes do ensino médio do município de Passos - MG. Ciênc Saúde Coletiva, 2011; 16(12):4745-4754.

11. Pinsky I,Sanches M, Zaleski M, Laranjeira R, Caetano R. Patterns of alcohol use among Brazilian adolescents. Rev Bras Psiquiatr. 2010; 32(3):242-49.

12. Vieira PC, Aerts DRGC, Freddo SL, Bittencout A, Monteiro L. Uso de álcool, tabaco e outras drogas por adolescentes escolares em município do Sul do Brasil. Cad Saúde Pública. 2008; 24(11):2487-98.

13. Cardoso LRD, Malbergier A. A influência dos amigos no consumo de drogas entre adolescentes. Estud Psicol (Campinas). 2014; 31(1):65-74.
14. Sanchez ZM, Martins SS, Opaleye ES, Moura YG, LocateIli DP, Noto AR. Social factors associated to binge drinking: a cross-sectional survey among Brazilian students in private high schools. BMC public health. 2011; 11:201.

15. Malta DC. et al. Família e proteção ao uso de tabaco álcool e drogas em adolescentes, Pesquisa Nacional de Saúde dos Escolares. Rev Bras Epidemiol. 2011; 14(3):166-177.

16. Locatelli D, Sanchez Z, Opaleye E, Carlini C, Noto A Socioeconomic influences on alcohol use patterns among private school students in São Paulo. Rev Bras de Psiquiatr. 2012; 34(2):193-200.

17. Dunn MS. Association between physical activity and substance use behaviors among high school students participating in the 2009 Youth Risk Behavior Survey. Psychol rep. 2014; 114(3):675-85.

18. Kwan M, Bobko S, Faulkner G, Donnelly P Cairney J. Sport participation and alcohol and illicit drug use in adolescents and young adults: a systematic review of longitudinal studies. Addict beh. 2014; 39(3):497-506

19. López Villalba FJ, Rodríguez García PL, García Cantó E, Pérez Soto JJ. Relationship between sport and physical activity and alcohol consumption among adolescents students in Murcia (Spain). Arch Argent Pediatr. 2016;114(2):101-6.

20. Kopp M, Burtscher M, Kopp-Wilfling P, Ruedl G, Kumnig M, Ledochowski L, Rumpold G. Is There a Link Between Physical Activity and Alcohol use? Subst Use Misuse. 2015; 50(5):546-51.

21. Dodge T, Clarke P, Dwan R. The Relationship Between Physical Activity and Alcohol Use Among Adults in the United States. Am J Health Promot. 2017; 31(2):97-108.

22. Lisha NE, Sussman S. Relationship of high school and college sports participation with alcohol, tobacco, and illicit drug use: A review. Addict Behav. 2010; 35(5):399-407.

23. Guedes DP, Lopes CC. Validação da versão brasileira do Youth Risk Behavior Survey 2007. Rev Saúde Pública. 2010; 44(5):840-850.

24. Zamboanga BL, Ham SH, Olthuis JV, Martens MP, Grossbard JR, Tyne KV. Alcohol expectancies and risky drinking behaviors among high school athletes: "l'd rather keep my head in the game". Prev Sci. 2012; 13(2): 140-49.

25. Granville-Garcia AF. et al. Alcohol consumption among adolescents: attitudes, behaviors and associated factors. Ciênc Saúde Coletiva. 2014; 19(1):7-16.

26. Zeitoune RCG. et al. O conhecimento de adolescentes sobre drogas lícitas e ilícitas: uma contribuição para a enfermagem comunitária. Esc Anna Nery. 2012; 16(1): 57-63. 
27. Bezerra J, Lopes AS, Hardman CM, Tassitano RM, Tenório MC, Barros MVG. Consumo de bebidas alcoólicas e tabagismo: associação com inatividade física no lazer e comportamento sedentário. Rev Andal Med Deporte. 2015; 8(1):1-6.

28. Noal RB, Menezes, AMB, Araújo CL, Hallal PC. Experimental use of alcohol in early adolescence: the 11-year follow-up of the 1993 Pelotas (Brazil) birth cohort study. Cad Saúde Pública, 2010; 26(10):1937-1944.

29. Hallal PC. et al. Global physical activity levels: surveillance progress, pitfalls, and prospects. Lancet. 2012; 380(9838):247-57.
30. Farias Júnior JC. et al. Comportamentos de risco à saúde em adolescentes no Sul do Brasil: prevalência e fatores associados. Rev Panam Salud Pública. 2009; 25(4):344-352.

\section{CORRESPONDÊNCIA}

Ana Carolina Rodarti Pitangui de Araújo

Colegiado de Fisioterapia -

Universidade de Pernambuco - UPE

Campus Petrolina - BR 203, Km 2 s/n Cidade Universitária CEP: 56328-903 - Petrolina - PE

E-mail: carolina.pitangui@upe.br 